\title{
Antiallodynic effect of intrathecal epigallocatechin-3-gallate due to suppression of reactive oxygen species
}

\author{
Sang Soon $\mathrm{An}^{1}$, Yeo Ok Kim², Cheon Hee Park ${ }^{1}$, Hai Lin ${ }^{2,3}$, and Myung Ha Yoon ${ }^{2,3}$ \\ Department of Anesthesiology and Pain Medicine, ${ }^{1}$ Gwangju Christian Hospital, ${ }^{2}$ Chonnam National University, Medical School, \\ ${ }^{3}$ Center for Creative Biomedical Scientists at Chonnam National University, Gwangju, Korea
}

Background: Green tea modulates neuropathic pain. Reactive oxygen species (ROS) are suggested as a key molecule in the underlying mechanism of neuropathic pain in the spinal cord. We examined the effect of epigallocatechin-3-gallate (EGCG), the major catechin in green tea, in neuropathic pain and clarified the involvement of ROS on the activity of EGCG.

Methods: Neuropathic pain was induced in male Sprague-Dawley rats by spinal nerve ligation (SNL). A polyethylene tube was intrathecally located. Nociceptive degree was estimated by a von Frey filament and expressed as a paw withdrawal threshold (PWT). To determine the role of ROS on the effect of EGCG, a free radical donor (tert-BuOOH) was pretreated before administration of EGCG. ROS activity was assayed by xanthine oxidase (XO) and malondialdehyde (MDA).

Results: SNL decreased the PWT compared to sham rats. The decrease remained during the entire observation period. Intrathecal EGCG increased the PWT at the SNL site. Intrathecal tert-BuOOH significantly decreased the effect of EGCG. The levels of both XO and MDA in the spinal cord were increased in SNL rats compared to sham. Intrathecal EGCG decreased the level of XO and MDA.

Conclusions: EGCG may reduce neuropathic pain by SNL due to the suppression of ROS in the spinal cord. (Korean J Anesthesiol 2014; 67: 123-128)

Key Words: EGCG, Neuropathic pain, ROS, Spinal cord.

Received: January 8, 2014. Revised: 1st, April 23, 2014; 2nd, June 9, 2014. Accepted: June 9, 2014.

Corresponding author: Myung Ha Yoon, M.D., Ph.D., Department of Anesthesiology and Pain Medicine, Chonnam National University, Medical School, 42, Jebong-ro, Dong-gu, Gwangju 501-757, Korea. Tel: 82-62-220-6893, Fax: 82-62-232-6294, E-mail: mhyoon@chonnam.ac.kr (c) This is an open-access article distributed under the terms of the Creative Commons Attribution Non-Commercial License (http:// creativecommons.org/licenses/by-nc/3.0/), which permits unrestricted non-commercial use, distribution, and reproduction in any medium, provided the original work is properly cited. 


\section{Introduction}

Chronic neuropathic pain develops directly because of a lesion or indirectly due to disease in the somatosensory system [1]. Although the incidence of neuropathic pain has been gradually increasing, therapeutic options remain limited because of the undetermined pathogenesis. Recently, the role of reactive oxygen species (ROS) has been emphasized in the generation of neuropathic pain $[2,3]$. Several lines of evidence indicate that well-known antioxidants including phenyl $N$-tert-butylnitrone (PBN), 5,5-dimethyl-1-pyrroline-N-oxide (DMPO), 4-hydroxy2,2,6,6-tetramethylpiperidine- $N$-oxyl (TEMPOL), and vitamin $\mathrm{E}$ are effective in relief of neuropathic pain in animal models [3-8]. Compounds that are able to inhibit ROS may be a good candidate for neuropathic pain therapy.

Green tea has been widely used as a beverage for a long time [9] and more recently has been explored in chronic disease therapy because of its antioxidant, anti-inflammatory and antimicrobial characteristics [10-12]. Epigallocatechin-3-gallate (EGCG) is the most active catechin in green tea and this major polyphenolic compound constitutes $30 \%$ of the dry leaf weight in the Camellia sinensis plant [13]. Previous animal studies have shown the antinociceptive effects of green tea extract in diabetic neuropathic pain and inflammatory hyperalgesia $[14,15]$. Intrathecal administration of EGCG attenuates neuropathic pain in a rat model through the blockade of nitric oxide synthase (NOS) [16]. However, the role of ROS on the effect of EGCG has been unresolved.

The purposes of this study were to test the effect of intrathecal EGCG in spinal nerve ligation (SNL)-induced neuropathic pain and to determine the contribution of ROS on the effect of EGCG at the spinal level.

\section{Materials and Methods}

\section{Animal preparation}

All of the experimental procedures and protocols were reviewed and approved by the Institutional Animal Care and Use Committee of University. Male Sprague-Dawley rats weighing 100-200 g were housed in a plastic cage with standard facilities $\left(22-23^{\circ} \mathrm{C}\right.$ room temperature, $12 / 12 \mathrm{~h}$ light/dark cycle with free access to water and food).

\section{Neuropathic pain modeling}

Makeup of neuropathic pain was done by lumbar SNL of rat, as described before [17]. Under sevoflurane in oxygen anesthesia, the left L5 and L6 spinal nerves were exposed after skin incision and dissection of surrounding structures. Then both nerves were tightly ligated with a 6-0 silk and the skin of rats was closed with a 3-0 silk. Sham operation was done except for SNL. After arousal from the anesthesia, rats were returned to vivarium for a recovery period of 5 days. SNL or the sham rats were examined for the development and maintenance of neuropathic pain for 21 days. Rats showing less than $4 \mathrm{~g}$ of the paw withdrawal threshold (PWT) in response to mechanical stimulation were classified as neuropathic pain state.

\section{Intrathecal catheterization}

For drug administration, a polyethylene tube (PE-10) was placed into the intrathecal space of rat under sevoflurane in oxygen anesthesia, as described before [18]. PE-10 was inserted through a punctural orifice of the atlantooccipital dura and was progressed downward to the lumbar enlargement. The proximal tip of the catheter was fixed in the scalp and was plugged with 30 gauge wire. The skin was closed with a 3-0 silk and rats recovered from anesthesia. Rats showing a postsurgical motor dysfunction were immediately euthanized with an overdose of sevoflurane. Normal rats were comfortably resided in a vivarium for 5 days.

\section{Drugs}

EGCG and tert-BuOOH were purchased from Sigma-Aldrich (St. Louis, MO, USA). The drugs were separately dissolved in $0.9 \%$ saline and were intrathecally injected in a volume of $10 \mu \mathrm{l}$ with a hand-driven, gear-operated syringe pump.

\section{Assessment of mechanical allodynia}

PWT to mechanical stimulation was measured as described before [19]. von Frey filament $(0.4,0.7,1.2,2.0,3.6,5.5,8.5$ and $15 \mathrm{~g})$ stimulation was done to the plantar surface of the paw of rats for $5 \mathrm{~s}$ with enough force to crook them. Abrupt withdrawal of foot during application of filament was reflected a positive response. In the positive case, the lighter filament was applied, otherwise the heavier one used. The cut-off value, in which no withdrawal response was seen, was at $15 \mathrm{~g}$ stimulation.

\section{Experimental protocol}

The behavioral effect of the saline and EGCG was observed 10 days after SNL. Rats were studied once. The researchers were blinded to the doses of EGCG to avoid subjective bias. Rats showing mechanical allodynia (PWT less than $4 \mathrm{~g}$ ) were included in the experiments. For testing, each rat was put into a transparent chamber with a wire mesh floor underneath, and was allowed to adapt to the chamber for $30 \mathrm{~min}$. Control thresh- 
old values were measured at immediately before the delivery of drugs.

\section{Effects of EGCG and reversal effects of free radical donor}

The behavioral effects of intrathecal saline and EGCG $(1,3$, $10 \mu \mathrm{g})$ were evaluated at $15,30,60,90$, and $120 \mathrm{~min}$ after injection. Next, to determine the involvement of ROS on the effect of EGCG, intrathecal tert-BuOOH (free radical donor, $10 \mu \mathrm{g}$ ) was administered 10 min before intrathecal delivery of EGCG. The dose of tert-BuOOH was selected from a preliminary study, which did not affect the PWT in SNL rats.

\section{Assays of ROS activity}

\section{Xanthine oxidase (XO) assay}

Spinal cord XO activity was assayed based on the multistep enzymatic reaction with the corresponding substrate using commercial kit (Cayman Chemical, Ann Arbor, MI, USA). The fluorescence of the sample was detected at an excitation wavelength of $535 \mathrm{~nm}$ and an emission wavelength of $590 \mathrm{~nm}$ in a XPS fluorescence microplate reader (Gemini, San Francisco, CA, USA). The enzyme activity was calculated per mg protein.

\section{Malondialdehyde (MDA) assay}

MDA is an end product of peroxidative decomposition of polyenic fatty acids in the lipid peroxidation process and its accumulation in tissues is indicative of the extent of lipid peroxidation. Tissue MDA levels were determined using a method based on reaction with thiobarbituric acid. Two hundred fifty microliters of the spinal cord protein samples was added to $35 \mu \mathrm{l}$ of DW and $25 \mu \mathrm{l}$ of $8.1 \%$ sodium dodecyl sulfate $190 \mu \mathrm{l}$ of the thiobarbituric acid reagent ( $0.8 \%$ thiobarbituric acid in $20 \%$ acetic acid) to precipitate protein. Then, the samples were heated in boiling water for $40 \mathrm{~min}$. After cooling, an equal volume (500 $\mu$ l of sample) of $n$-butanol/pyridine $(15: 1)$ was added to each test tube and mixed. The mixture was centrifuged at $12,000 \mathrm{rpm}$ for $10 \mathrm{~min}$ at room temperature. The absorbance of the organic layer was read at $532 \mathrm{~nm}$ in a $300 \mu \mathrm{l}$ suspension using a Versamax microplate reader (Molecular Devices, Sunnyvale, CA, USA). MDA concentrations were calculated by comparing the absorbance values of the samples with those of standard MDA solutions. The MDA concentrations are expressed in nmoles per gram of wet weight tissue.

\section{General behavior}

The motor function effects of EGCG $(n=3)$ and tert-BuOOH $(n=3)$ at the highest injected doses were evaluated in an open space. The placing-stepping reflex, induced by drawing the dorsum of hind paw across the edge of the table, was normal if rats try to put their paws forward into a position for walking. The righting reflex, evoked by placing the rat horizontally with its back on the table, was normal if rats produce immediate, coordinated twisting of the body into an upright position. The presence of both reflexes was considered as a normal.

\section{Statistical analyses}

All data are expressed as mean \pm SEM. Time course and dose-response data are presented as PWT or as the percent of maximum possible effect (\%MPE) to mechanical stimulation. PWT data were converted to \%MPE, according to the formula: $\% \mathrm{MPE}=[$ (post drug threshold - post-injured baseline threshold) / (cutoff threshold - post-injured baseline threshold)] $\times$ 100. Dose-response data were analyzed using one-way analysis of variance followed by Scheffés post hoc test. Antagonism of EGCG and the concentration of 5-HT were analyzed using an unpaired t-test. $\mathrm{P}<0.05$ was accepted as statistical significance.

\section{Results}

As the motor function index, both the placing-stepping reflex and the righting reflex were normally present after intrathecal administration of EGCG and tert-BuOOH at the injected doses. The PWT was significantly decreased compared to baseline and was persistent during the 21 days of observation in SNL rats (Fig. 1). The baseline PWT measured before surgery was $12.8 \pm 1$ in SNL and sham rats. Sham surgery did not affect the PWT, but SNL

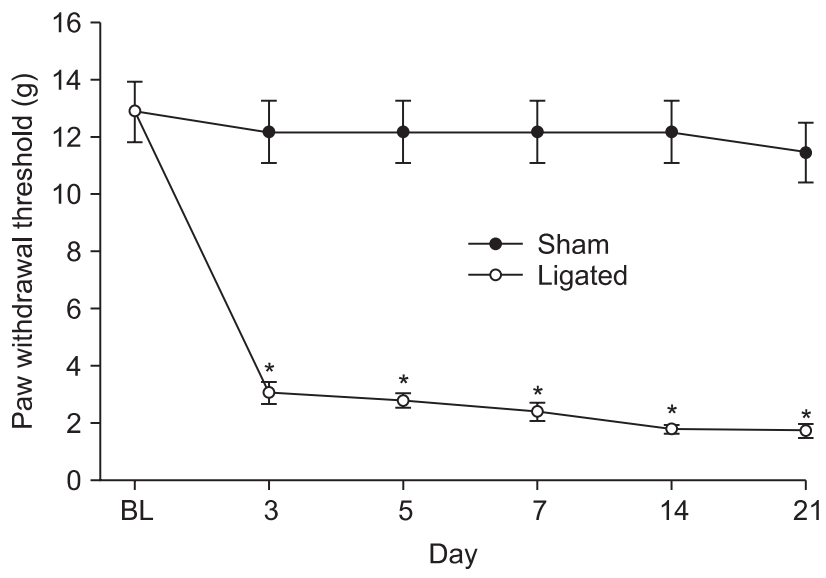

Fig. 1. Paw withdrawal threshold (PWT) to von Frey filament application in sham and spinal nerve ligation (SNL) rats. Data of the PWT are presented as weight $(\mathrm{g})$. Each line represents mean \pm SEM of five rats. BL: baseline PWT measured before SNL. SNL significantly decreased the PWT in ligated site, ${ }^{*} \mathrm{P}<0.001$, vs. sham. 
A

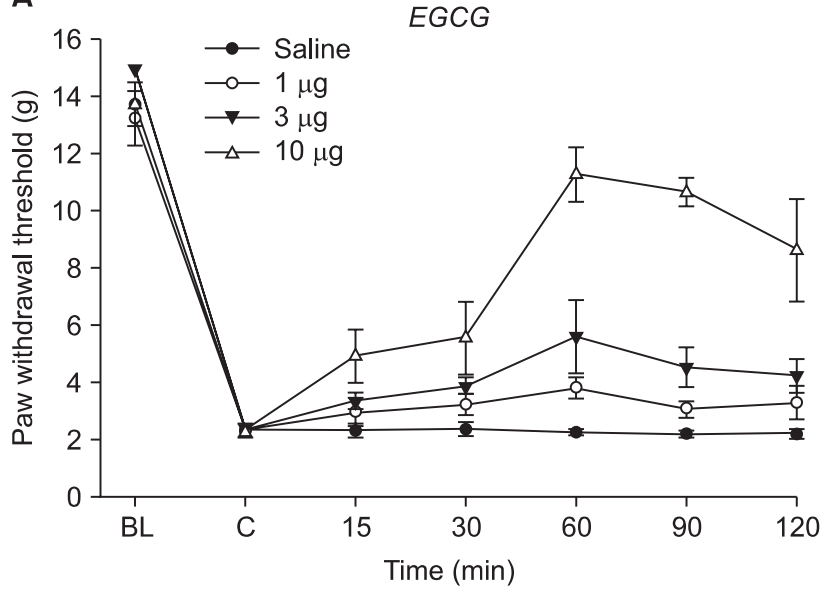

B

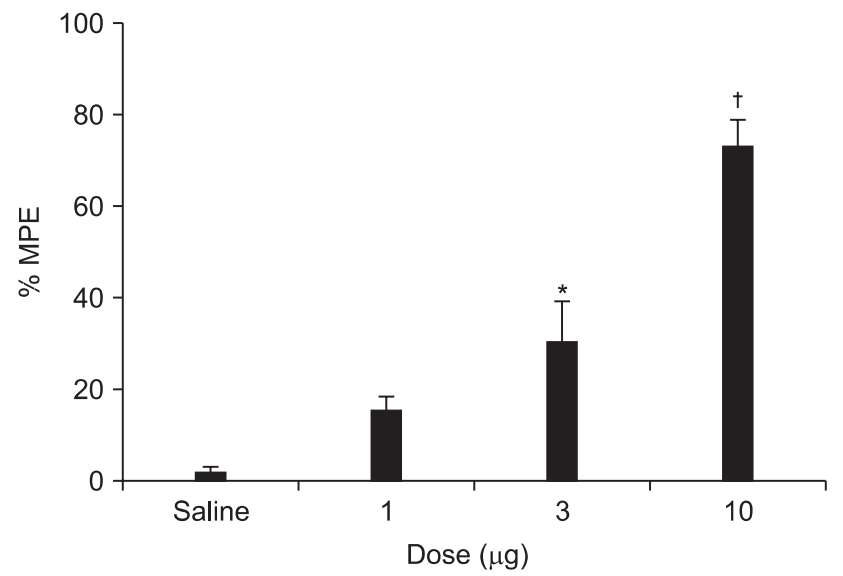

Fig. 2. Data of the PWT are presented as weight (A) or percentages of maximal possible effect (\%MPE, B). Effects of intrathecal saline and epigallocatechin-3-gallate (EGCG) on the paw withdrawal threshold (PWT) to von Frey filament application in spinal nerve ligation (SNL) rats. Data of the PWT are presented as weight (g). Each line represents mean \pm SEM of five or six rats. BL: baseline PWT measured before SNL. C: control PWT measured immediately before EGCG administration. Intrathecal EGCG dose-dependently increased the PWT, ${ }^{*} \mathrm{P}<0.05,{ }^{\dagger} \mathrm{P}<0.001$, vs. saline.

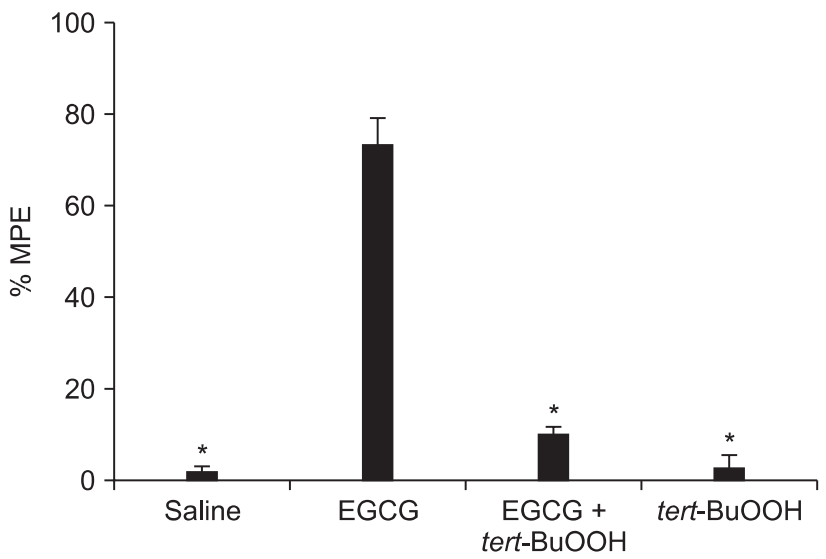

Fig. 3. Effects of tert-BuOOH $(10 \mu \mathrm{g})$ on the antiallodynic effect of epigallocatechin-3-gallate (EGCG, $10 \mu \mathrm{g}$ ) in spinal nerve ligation (SNL) rats. Data are presented as \%MPE. Each bar represents mean \pm SEM of five rats. Intrathecal tert- $\mathrm{BuOOH}$, administered $10 \mathrm{~min}$ before the injection of EGCG decreased the antiallodynic effect of intrathecal EGCG, $*$ P $<0.001$, vs. EGCG.

surgery decreased the PWT compared to sham group (Fig. 1).

Both the baseline PWT measured before SNL and control PWT measured immediately before EGCG administration did not differ from each other (Fig. 2). Intrathecal EGCG dosedependently increased the PWT at the SNL site. The MPE of EGCG at $10 \mu$ g was approximately $73 \%$ (Fig. 2).

Intrathecal tert-BuOOH antagonized the antiallodynic effect of EGCG (Fig. 3). tert-BuOOH itself did not affect the paw withdrawal threshold.

The levels of XO and MDA in spinal cord were significantly elevated in the SNL rats compared to the sham rats. Intrathecal EGCG decreased the levels of XO and MDA in the spinal cord of ligated rats (Fig. 4).

\section{Discussion}

In the present study, the effect of EGCG on neuropathic pain induced by SNL was investigated. Intrathecal EGCG was active against neuropathic pain behavior. This antiallodynic effect of EGCG was antagonized by free radical donor. In addition, $\mathrm{XO}$ and MDA levels were considerably increased in the spinal cord of neuropathic rats. On the other hand, intrathecal EGCG significantly reduced the levels of $\mathrm{XO}$ and MDA. These findings suggest that EGCG is effective for the relief of neuropathic pain, and that the activity of EGCG is mediated through the suppression of ROS in the spinal cord.

Accumulating evidence suggests that green tea polyphenols, especially the most predominant green tea catechin, EGCG, have beneficial effects on health [13]. EGCG has many preventive, therapeutic and protective effects on cancer, cardiovascular diseases, and neurodegenerative diseases [12] based on a variety of activities including antioxidant property due to scavenging of free radicals of ROS [20,21]. EGCG also has iron-chelating activity [20].

Less is known about the effect of EGCG or green tea in nociceptive modulation compared to other medical areas. Moreover, the mechanism of action has not been definitely resolved. Several studies have provided some information about EGCG. Intraperitoneal injection of green tea extract reduced streptozotocininduced diabetic thermal hyperalgesia by inhibition of nitric oxide formation [14]. Oral administration of green tea decreases mechanical allodynia and hyperalgesia in chronic constriction injury-induced neuropathic pain [22]. Intraperitoneal green tea 
A

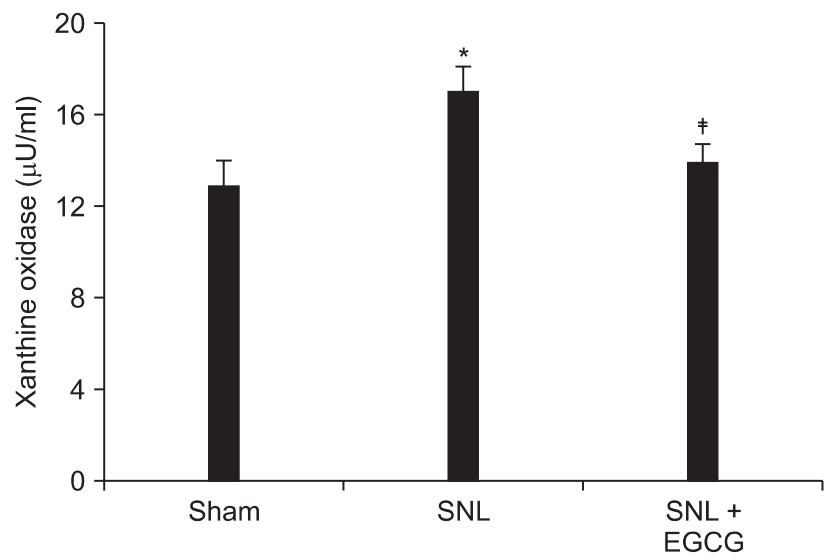

B

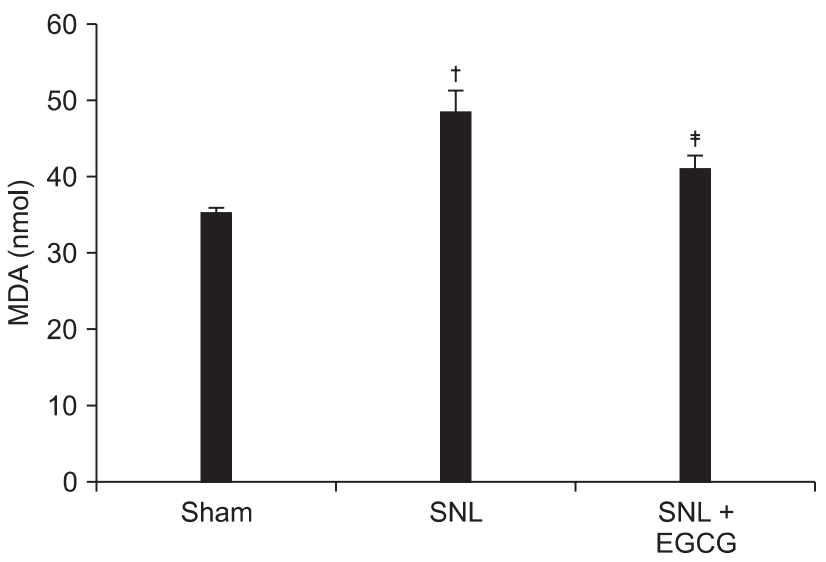

Fig. 4. Levels of xanthine oxidase (XO, A) and malondi aldehyde (MDA, B) in the spinal cord. Each bar represents mean \pm SEM of five rats. Both XO and MDA levels of spinal nerve-ligated rats were higher than those of naïve rats, ${ }^{*} \mathrm{P}<0.05,{ }^{\dagger} \mathrm{P}<0.01$, vs. naive. Intrathecal EGCG decreased the levels of $\mathrm{XO}$ and MDA, ${ }^{\ddagger} \mathrm{P}<0.05$, vs. SNL.

extract can significantly attenuate lipopolysaccharide-induced thermal and behavioral hyperalgesia by selective inhibition of cyclooxygenase-2 [15]. Intrathecal EGCG reduces mechanical allodynia evoked by SNL by inhibition of nitric oxide synthase [16]. Intrathecal EGCG increases mechanical and heat pain thresholds in chronic constriction injury-induced neuropathic pain by inhibition of toll-like receptor [23].

On the other hand, the antiallodynic effect of EGCG was reversed by free radical donor in the current study. These results indicate that ROS inhibition may contribute to the mechanism of EGCG in the spinal cord. ROS like superoxide and hydrogen peroxide are strong reactive molecules derived from $\mathrm{O}_{2}$. Although ROS are necessary for body physiology, excessive ROS provoke oxidative stress and lessens the normal roles of ROS, which leads to a deleterious damage to cell structures including lipids and membranes, proteins, and DNA [24,25]. Overproduction of ROS may be linked to the pathogenesis of several diseases including cancer, cardiovascular disease, atherosclerosis, hypertension, ischemia/reperfusion injury, diabetes mellitus, neurodegenerative diseases, and rheumatoid arthritis $[24,26]$. In particular, ROS are very critical molecules in the processing of signal transduction in the spinal cord [27]. Furthermore, elevated ROS are involved in the potentiation of synaptic excitability in the spinal dorsal horn [28]. Importantly, ROS have been implicated in the etiology of neuropathic pain $[2,3]$. Free radical scavengers and antioxidants significantly attenuate neuropathic pain [3-8]. Therefore, ROS may be involved in the mediation of nociceptive signaling in the dorsal horn of the spinal cord. However, the antiallodynic effect of EGCG was not reversed completely by free radical donor. That finding suggests that other moleclules besides ROS are also involved in the activity of EGCG. Although the underlying mechanisms of neuropathic pain mediated by ROS in the spinal cord are unknown, various pathways have been reported. ROS are involved in NMDA-receptor activation [2], p38 mitogen activated protein kinase phosphorylation [4], expression of some apoptotic genes like bax, apoptotic protease-activating factor-1 (apaf-1),and caspase-9 [6], reducing spinal GABA release [7], activation of ion channels like transient receptor potential ankyrin 1 and transient receptor potential vanilloid 1 channels [29], activation of calcium-calmodulin-dependent protein kinase II (CamKII) [30], and NOS activation in the spinal cord, which may contribute to the development of neuropathic pain. Furthermore, the present data showing the elevated levels of XO and MDA by neuropathic pain induction support the view that ROS are important molecules in neuropathic pain genesis.

There are some limitations to the present study. First, we just investigated the involvement of ROS on the neuropathic pain. The subjects of downstream of ROS in neuropathy and the effect of EGCG on that downstream have not been evaluated. Second, we assayed the ROS activity indirectly. Unfortunately, we did not use the electrophysiological recordings. Thus, further studies are required to consider these issues.

Taken together, the data indicate that intrathecal EGCG has an antiallodynic effect against neuropathic pain by SNL. This effect is antagonized by free radical donor. Additionally, intrathecal EGCG decreases elevated ROS induced by SNL in the spinal cord. Thus, EGCG may be a therapeutic option for neuropathic pain through the attenuation of ROS activity at the spinal level. 


\section{References}

1. Treede RD, Jensen TS, Campbell JN, Cruccu G, Dostrovsky JO, Griffin JW, et al. Neuropathic pain: redefinition and a grading system for clinical and research purposes. Neurology 2008; 70: 1630-5.

2. Gao X, Kim HK, Chung JM, Chung K. Reactive oxygen species (ROS) are involved in enhancement of NMDA-receptor phosphorylation in animal models of pain. Pain 2007; 131: 262-71.

3. Kim HK, Park SK, Zhou JL, Taglialatela G, Chung K, Coggeshall RE, et al. Reactive oxygen species (ROS) play an important role in a rat model of neuropathic pain. Pain 2004; 111: 116-24.

4. Lu R, Kallenborn-Gerhardt W, Geisslinger G, Schmidtko A. Additive antinociceptive effects of a combination of vitamin C and vitamin E after peripheral nerve injury. PLoS One 2011; 6: e29240.

5. Mao YF, Yan N, Xu H, Sun JH, Xiong YC, Deng XM. Edaravone, a free radical scavenger, is effective on neuropathic pain in rats. Brain Res 2009; 1248: 68-75.

6. Siniscalco D, Fuccio C, Giordano C, Ferraraccio F, Palazzo E, Luongo L, et al. Role of reactive oxygen species and spinal cord apoptotic genes in the development of neuropathic pain. Pharmacol Res 2007; 55: 158-66.

7. Yowtak J, Lee KY, Kim HY, Wang J, Kim HK, Chung K, et al. Reactive oxygen species contribute to neuropathic pain by reducing spinal GABA release. Pain 2011; 152: 844-52.

8. Kallenborn-Gerhardt W, Lu R, Syhr KM, Heidler J, von Melchner H, Geisslinger G, et al. Antioxidant activity of sestrin 2 controls neuropathic pain after peripheral nerve injury. Antioxid Redox Signal 2013; 19: 2013-23.

9. Weisbuerger JH. Tea and health: the underlying mechanisms. Proc Soc Exp Biol Med 1999; 220: 271-5.

10. Gaur S, Agnihotri R. Green tea: A novel functional food for the oral health of older adults. Geriatr Gerontol Int 2014; 14: 238-50.

11. Tao L, Forester SC, Lambert JD. The role of the mitochondrial oxidative stress in the cytotoxic effects of the green tea catechin, (-)-epigallocatechin-3-gallate, in oral cells. Mol Nutr Food Res 2014; 58: 665-76.

12. Zaveri NT. Green tea and its polyphenolic catechins: medicinal uses in cancer and noncancer applications. Life Sci 2006; 78: 2073-80.

13. Graham HN. Green tea composition, consumption, and polyphenol chemistry. Prev Med 1992; 21: 334-50.

14. Singal A, Anjaneyulu M, Chopra K. Modulatory role of green tea extract on antinociceptive effect of morphine in diabetic mice. J Med Food 2005; 8: 386-91.

15. Kaur S, Anurag A, Tirkey N, Chopra K. Reversal of LPS-induced central and peripheral hyperalgesia by green tea extract. Phytother Res 2005; 19: 39-43.

16. Choi JI, Kim WM, Lee HG, Kim YO, Yoon MH. Role of neuronal nitric oxide synthase in the antiallodynic effects of intrathecal EGCG in a neuropathic pain rat model. Neurosci Lett 2012; 510: 53-7.

17. Kim SH, Chung JM. An experimental model for peripheral neuropathy produced by segmental spinal nerve ligation in the rat. Pain 1992; 50: 355-63.

18. Yaksh TL, Rudy TA. Chronic catheterization of the spinal subarachnoid space. Physiol Behav 1976; 17: 1031-6.

19. Chaplan SR, Bach FW, Pogrel JW, Chung JM, Yaksh TL. Quantitative assessment of tactile allodynia in the rat paw. J Neurosci Methods 1994; 53: 55-63.

20. Weinreb O, Amit T, Mandel S, Youdim MB. Neuroprotective molecular mechanisms of (-)-epigallocatechin-3-gallate: a reflective outcome of its antioxidant, iron chelating and neuritogenic properties. Genes Nutr 2009; 4: 283-96.

21. Mandel SA, Avramovich-Tirosh Y, Reznichenko L, Zheng H, Weinreb O, Amit T, et al. Multifunctional activities of green tea catechins in neuroprotection. Modulation of cell survival genes, iron-dependent oxidative stress and PKC signaling pathway. Neurosignals 2005; 14: 4660.

22. Renno WM, Saleh F, Klepacek I, Al-Khaledi G, Ismael H, Asfar S. Green tea pain modulating effect in sciatic nerve chronic constriction injury rat model. Nutr Neurosci 2006; 9: 41-7.

23. Kuang X, Huang Y, Gu HF, Zu XY, Zou WY, Song ZB, et al. Effects of intrathecal epigallocatechin gallate, an inhibitor of Toll-like receptor 4, on chronic neuropathic pain in rats. Eur J Pharmacol 2012; 676: 51-6.

24. Valko M, Leibfritz D, Moncol J, Cronin MT, Mazur M, Telser J. Free radicals and antioxidants in normal physiological functions and human disease. Int J Biochem Cell Biol 2007; 39: 44-84.

25. Lander HM. An essential role for free radicals and derived species in signal transduction. FASEB J 1997; 11: 118-24.

26. Dröge W. Free radicals in the physiological control of cell function. Physiol Rev 2002; 82: 47-95.

27. Kennedy KA, Sandiford SD, Skerjanc IS, Li SS. Reactive oxygen species and the neuronal fate. Cell Mol Life Sci 2012; 69: 215-21.

28. Lee KY, Chung K, Chung JM. Involvement of reactive oxygen species in long-term potentiation in the spinal cord dorsal horn. J Neurophysiol 2010; 103: 382-91.

29. Nishio N, Taniguchi W, Sugimura YK, Takiguchi N, Yamanaka M, Kiyoyuki Y, et al. Reactive oxygen species enhance excitatory synaptic transmission in rat spinal dorsal horn neurons by activating TRPA1 and TRPV1 channels. Neuroscience 2013; 247: 201-12.

30. Gwak YS, Hassler SE, Hulsebosch CE. Reactive oxygen species contribute to neuropathic pain and locomotor dysfunction via activation of CamKII in remote segments following spinal cord contusion injury in rats. Pain 2013; 154: 1699-708. 